\title{
Application of Structural Design in Advanced Clothing
}

\author{
Xiaoli Cheng \\ Guangdong University of Science \& Technology, Dongguan, Guangdong, China, 523083
}

Keywords: structural design; advanced garment; comprehensive; process design

\begin{abstract}
In fact, the structural design is the overall design and finished product effect of the garment design. Garment structure design is a comprehensive work, which not only requires the designer to master a certain amount of knowledge, but also needs the designer to have a flexible, innovative thinking, in order to fully play the role of structural design in the design of advanced garments. The design of ready-to-wear garments must rely on structural design to ensure their effectiveness. It can be said that structural design plays an important role in advanced garment design. Its importance is irreplaceable. For a high-end garment, modeling, structural design and process design are the three most important aspects of the design process. Among them, structural design has a special role and significance, that is, it is an extension and development of style design, and it is also the preparation and foundation of process design. The research in this paper is mainly about the application of structural design in high-end garments.
\end{abstract}

\section{Introduction}

Structural design is the key link that best embodies the new design concept. Advanced garment design is a structural overall design that requires a specific space and is completed with the overall concept. It is the final procedure for dealing with the relationship between structure and structure. From this we can see that structural design has a certain importance in advanced garment design. In this article, the author roughly talks about his understanding of the application of structural design in high-grade garments.

\section{The Core Role of Structural Design}

Clothing structure design is a discipline that studies and discusses the relationship between human body structure and clothing style from the perspective of plastic arts. In the costume designer's discipline, the three aspects of modeling design, structural design and process design are all organically connected and inseparable. The former is based on the target of service, establishing the final effect of the costume model; the latter is the process of completing the modeling structure of the sewing process.

The structural design is one of the components of the high-end garment design. The content is to decompose the entire garment contour shape and various parts of the stereotypes defined by the high-grade garments, and to show the flat effect of the garments in the form of drawings, which is not allowed during the process of garment design revision. the missing part. Practice has proved that structural design can effectively, reasonably and quickly correct the unreasonable structural relationship of garment design, thus ensuring the perfectness and effectiveness of garment production. From the perspective of the composition of advanced garment design, structural design is an extension and development of style design work ${ }^{[1]}$. At the same time, it is the starting point and foundation of process design and has a certain core role. It can be seen that the structural design has an irreplaceable role for high-grade garments.

\section{Structure Design Content}

Clothing plate sample. Basics of clothing, various skirts, men's and women's trousers, jeans, casual pants, men's and women's shirts, men's and women's suits, tunic, women's fashion, raglan 
sleeves, cheongsam, men's and women's coats, dresses, jackets, knitted patterns, children's wear patterns, Individual tailoring, pattern making techniques ${ }^{[2]}$.

Fashion industry making. Apparel industrial structure design, industrial look, sample, see the single sample and skills, such as various types of skirts, pants, women's fashion, cotton, women's trench coats, dresses, women's coats, sportswear, raglan sleeves, men's jackets, Knitted shirts, all kinds of fashion, men's and women's denim shirts. Various types of apparel industry grading, the latest collar type, sleeve type, version type method and skills.

Clothing technology. Car control skills, more than a dozen craft sewing methods, eight types of angle. And a variety of bags, collars, zippers, trousers, split ends and the entire skirt, trousers, fashion and other craftsmanship and other methods of operation.

History of clothing development. History of ancient Chinese clothing, Western clothing history, modern clothing, masterpieces of Chinese and foreign masterpieces, etc.

Clothing sketch. The basic factors and means of sketch modeling, perspective change laws and applications, the significance of painting geometric shapes, studying the perspective of straight lines and curves, still life sketches, plaster sketches, character sketches, human sketches, sketches, etc.

Plane composition. Mastering the midpoint, line, and face of clothing is the most basic element, the meaning and classification of composition, the common method of composition, and the opening of composition. Such as cross-belt, layered, full-scale, scattered, grid, fit, symmetrical, balanced, radial, etc., and in apparel design.

Stereoscopic composition. Three-dimensional modeling elements, three-dimensional aesthetic principles, three-dimensional composition of the production process, the three-dimensional composition of the application design, body composition of production training, three-dimensional composition of clothing styles.

Color composition and clothing color. Color concept, three elements, color mixing and color contrast, color psychology, structural color, clothing and color, clothing color color matching principle, overall design and source, fashion color

Fashion painting techniques. Quiet, dynamic and various angles of painting and proportions, head, hand and foot painting, various clothing styles, clothing patterns, gouache, watercolors, colored lead and other coloring techniques, various fabric textures, performance of various types of clothing Techniques, coloring techniques.

Fashion design. Design process for creative equipment, classification, characteristics, operation of garment design, operation of branded apparel and design rooms, design of garment parts (collar, sleeve, bag)

Pattern design. The source of pattern modeling materials, various costume pattern designs, pattern shape expressions, pattern design methods and natural pattern shape expressions, pattern collection, and costume pattern design techniques.

Clothing materials. Performances and characteristics of cotton, linen, wool, silk, chemical fiber, leather, etc., structural characteristics and properties of woven fabrics, knitted fabrics, and non-woven fabrics, simple identification methods for fibers, and garment accessories.

\section{Application of Structural Design in Advanced Clothing}

In general, observing the human body model, the basic structural lines determined in the human body model are derived from obvious bumpy points (Figure 1), and the horizontal transitions are evident on the bust line, waist line, and hip line. Here is the location where the circumference reaches the extreme value, as well as the dividing line that changes the circumference ${ }^{[3]}$. Due to the limitation of production costs, it is not advisable to design too many dividing lines. 

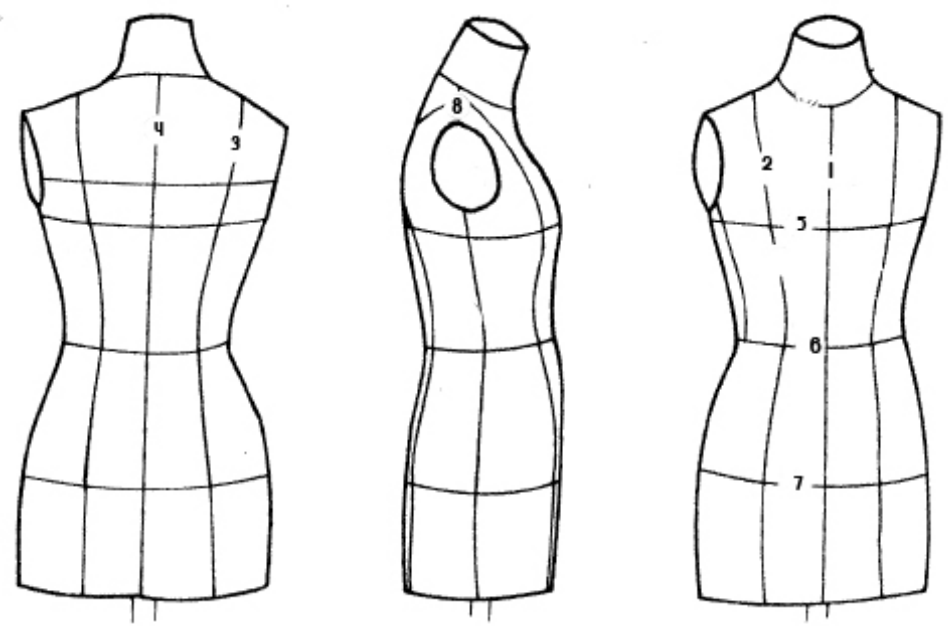

Figure 1 Basic structure line

\subsection{Application of segmentation and its application in advanced clothing.}

The dividing line of a garment refers to a line that is embodied in each spliced part of the garment and constitutes the entire garment. Mainly include the threshold line, provincial line, pleat line, bottom cyclone and the body of the sewing and so on. Segmentation itself has the value of recognizing the new space and applying the new space. The proper segmentation is for better integration and reorganization. Through segmentation, garments can be updated and make better use of space. Clothing is ultimately worn on the human body, so the dividing line of clothing has a close relationship with the body's physical characteristics. Segmentation modeling should follow the following principles: (1) The division line design should be based on the basic function of the structure. The basic function of the structure is to make the garment comfortable, convenient and attractive. Therefore, the design of split lines is not arbitrary.

This article takes a garment with a length above hips as an example to create a basic type of princess clothing. According to the principle of three-dimensional transformation to plane, in the most obvious position of human body turning, the woman body is decomposed into eight faces, and pieces of clothing for each face are made separately, then placed in the same plane space, and connected into four pieces of clothing, as shown in the figure. 2 shows. This segmented design makes the garments fit together, has a beautiful shape, is rich in three-dimensional, and has a strong plasticity.

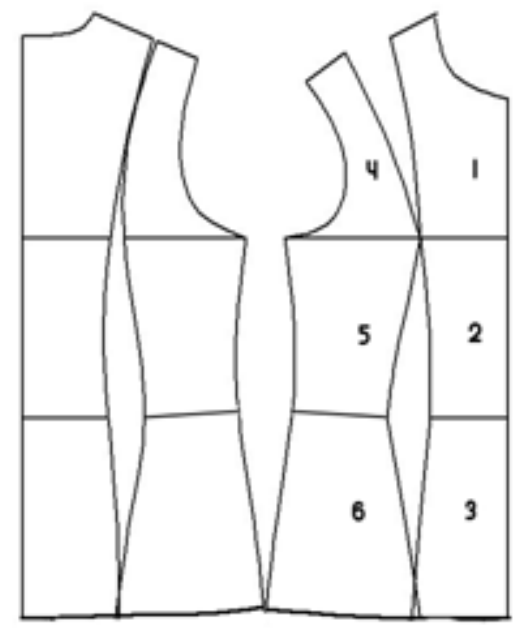

Figure 2 Woman body solution

\subsection{Application of structural design of stereoscopic thinking in advanced clothing.}

The garment structure design is based on the human body and the human body is a specific three-dimensional body. Therefore, it is necessary to deeply understand the relationship between the garment structure and the human body surface. In nature, "straight and circular lines and their various 
combinations and changes can define and describe any visible object", and can produce infinitely varied forms. If we consider the surface of the human body as a shell consisting of a large number of tightly arranged thin lines, then the garment is the packaging of this shell and it is also composed of numerous dense lines ${ }^{[4]}$. According to this view, although the appearance of the human body is an irregularly changing curved surface, we can still find the turning lines that are more obvious on the human body based on the turning of bones and muscles, and provide a reference for converting the flat cloth into a three-dimensional garment.

\subsection{Ergonomic corrections.}

We use the three-dimensional cutting method to get the princess line segmented clothing piece shape, but also can not be directly applied to the cutting production of tight-fitting clothing. This is because people will change with the breathing, exercise and other activities, the surface area of the skin, based on Pieces made by static mannequins cannot adapt to the needs of the human body if they do not add relaxation. According to ergonomic calculations, the change in the chest circumference of a person's breath is about $4 \mathrm{~cm}$. Since this change is uniform, the amount of increase in the four pieces of clothing can be equal to $0.5 \mathrm{~cm}$ for each piece. Experiments have proved that this amount is more The degree of fit of finished garments to the human body has no significant effect.

However, for some of the more relaxed princess sewn garments, such as coats, coats, etc., the amount of relaxation should be considered. Because the design of the princess line is based on the dividing line designed by the body's turning line, once the dividing line is far from the turning line of the human body, it will lose the effect of fitting the human body ${ }^{[5]}$. The following figure 3 is a comparison chart of the front and back side garments processed on the basis of the basic clothing piece of the Princess line. According to the picture, it can be seen that the princess line above the waistline becomes straight, the waistline becomes smaller, and there is no change below the waistline. Analysis of change principle: The part of the waist line above the waist line shifted to the side seam, reducing the gradient of the contour of the garment. Reduced lateral tension caused by large provinces and eliminated diagonal wrinkles.

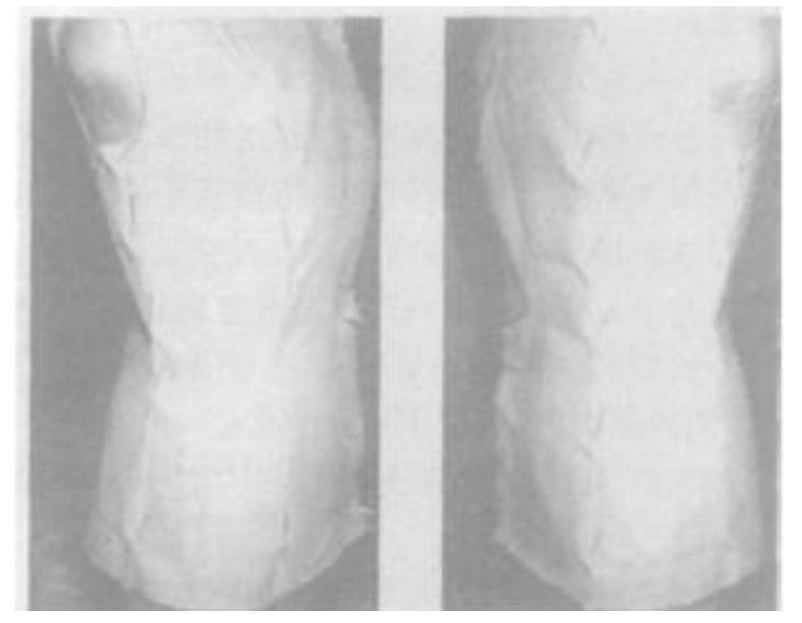

Figure 3 Comparison of front and rear garments

\section{Conclusion}

In summary, the human body is a specific three-dimensional body. The design of the garment structure cannot be separated from the three-dimensional thinking. The author mainly takes human body shape characteristics as the basic point, and uses the design of the princess's seam structure as the carrier to discuss the three-dimensional structure of the clothing. The three-dimensional design effect-two-dimensional swatch - the experimental process of three-dimensional clothing conversion, and the creation of the proposed method, while applying the three-dimensional thinking and method, combined with the characteristics of the fabric, such as the yarn to the details, the legislation, The organic combination of pattern-making techniques and fabric characteristics will create rich and 
colorful clothing shapes. This study hopes to provide a valuable reference for peers to further study the design of lines in clothing.

\section{References}

[1] Wenbin Zhang. Costume Technology (Structural Design Volume) [M ]1 Beijing: China Textile Press, 20011

[2] [Japan] Nakazawa Yu. Human body and clothing [M] 1 Beijing: China Textile Press, 20021

[3] Gu Yunfen. Application and structural design of split line on clothing[J]1Apparel Technology, 2000, (1): 36 391

[4] Cao Wenli; Xiu Yi. The parametric design model of garment pattern based on graph theory[J]; Journal of Beijing Institute of Clothing Technology (Natural Science);2008-01

[5] Jiang Zhilin. Analysis of how to transform costume effect maps into garments's plane structure map[J]; Fujian Tribune (Social Sciences Education Edition);2009-S1 